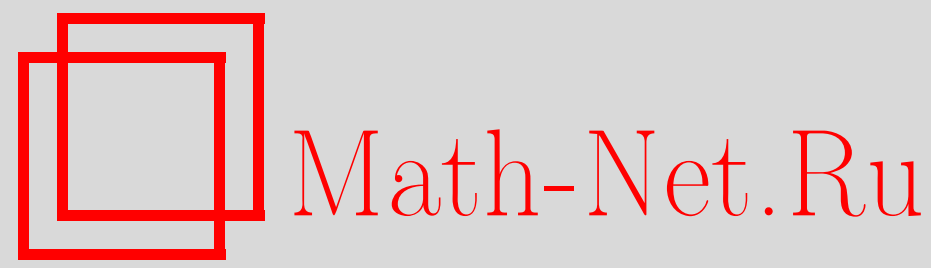

В. И. Скалыга, Аналоги неравенства братьев Марковых для полиномов на кубе в $\mathbb{R}^{m}$, Матем. заметки, 1996, том 60, выпуск 5, 783-787

DOI: https://doi.org/10.4213/mzm1894

Использование Общероссийского математического портала MathNet.Ru подразумевает, что вы прочитали и согласны с пользовательским соглашением

http://www . mathnet.ru/rus/agreement

Параметры загрузки:

IP : 54.162 .27 .143

26 апреля 2023 г., 12:33:40

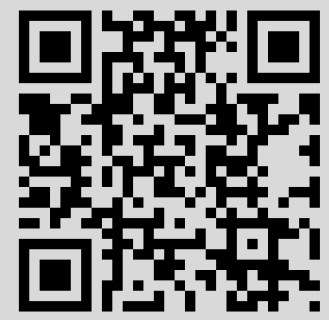




\section{АНАЛОГИ НЕРАВЕНСТВА БРАТЬЕВ МАРКОВЫХ ДЛЯ ПОЛИНОМОВ НА КУБЕ В $\mathbb{R}^{m}$}

\section{В.И. Скалыга}

1. Введение. Рассмотрим известное неравенство А. А. и В. А. Марковых для полиномов $n$-ой степени от одной переменной $[1$, c. 281$]$.

Если для полинома $P_{n}(x),\left|P_{n}(x)\right| \leqslant 1$ при $x \in[-1,1]$, то для тех же $x$ и $k$-ой производной $P_{n}^{(k)}(x)$ полинома $P_{n}(x)$ справедливо неравенство

$$
\left|P_{n}^{(k)}(x)\right| \leqslant \frac{n^{2}\left(n^{2}-1\right) \cdots\left(n^{2}-(k-1)\right)}{3 \cdots(2 k-1)}=T_{n}^{(k)}(1),
$$

где $T_{n}(x)=\cos (n \cdot \arccos x)$ - многочлен Чебышева.

Неравенство (1) при $k=1$ доказано А. А. Марковым, при $k \geqslant 2-$ В. А. Марковым.

В работе [2] неравенство А.А. Маркова с той же константой $n^{2}$ доказано для полиномов $n$-ой степени от $m$ переменных

$$
P_{n}(x)=P_{n}\left(x_{1}, \ldots, x_{m}\right)=\sum a_{i_{1}, \ldots, i_{m}} x_{1}^{i_{1}} \cdots x_{m}^{i_{m}} \quad\left(\sum_{j=1}^{m} i_{j} \leqslant n\right),
$$

рассматриваемых на единичном шаре в пространстве $\mathbb{R}_{l_{2}}^{m}$ с евклидовой нормой

$$
\|x\|_{l_{2}}=\left(\sum_{i=1}^{m} x_{i}^{2}\right)^{1 / 2}
$$

В работе [3] неравенство А. А. Маркова обобщено для выпуклых компактов $\mathbb{R}_{l_{2}}^{m}$ с константой $4 n^{2} / d$, где $d$ - диаметр максимального шара, содержашегося в компакте.

В настоящей работе приводятся аналоги неравенства (1) для полиномов вида (2) на кубе в пространстве $\mathbb{R}_{l_{2}}^{m}$ и $\mathbb{R}_{l_{1}}^{m}$. Норма в $\mathbb{R}_{l_{1}}^{m}$ задается следующей формулой:

$$
\|x\|_{l_{1}}=\sum_{i=1}^{m}\left|x_{i}\right|
$$

2. Вспомогательные леммы. В последующих двух леммах будут рассматриваться полиномы $P_{n}(x)$ от одной переменной $x$.

Лемма 1. Пусть для полинома $P_{n-2}(x)$ при $x \in[-1,1]$

$$
\left|\left(1-x^{2}\right) P_{n-2}(x)\right| \leqslant 1 \text {. }
$$

Тогда

$$
\max _{x \in[-1,1]}\left|P_{n-2}(x)\right| \leqslant \frac{n^{2}-1}{3} .
$$

Работа вьполненапри финансовой поддержке Российского фонда фундаментальных исследований, грант № 93-011-16021. 
ДоКАЗАТЕЛЬСТво. Пусть $U_{n-1}(x)=T_{n}^{\prime}(x) / n=\sin n \nu / \sin \nu$, где $\nu=\arccos x$. Напишем для $P_{n-2}(x)$ интерполяционную формулу Лагранжа

$$
\begin{aligned}
P_{n-2}(x) & =\sum_{i=1}^{n-1} \frac{U_{n-1}(x) P_{n-2}\left(x_{i}\right)}{U_{n-1}^{\prime}\left(x_{i}\right)\left(x-x_{i}\right)} \\
& =\frac{1}{n} \sum_{i=1}^{n-1}(-1)^{i-1} \frac{\left(1-x_{i}^{2}\right) P_{n-2}\left(x_{i}\right) U_{n-1}(x)}{x-x_{i}}
\end{aligned}
$$

где $x_{i}=\cos (i \pi / n)(i=1,2, \ldots, n-1)$ - нули полинома $U_{n-1}(x)$. Пусть $x_{n-1} \leqslant x \leqslant x_{1}$. Тогда

$$
1-x^{2} \geqslant \sin ^{2} \frac{\pi}{n} \geqslant \frac{4}{\pi^{2}} \cdot \frac{\pi^{2}}{n^{2}}=\frac{4}{n^{2}} .
$$

Из (3) и (4) для этих $x$ следует неравенство

$$
\left|P_{n-2}(x)\right| \leqslant \frac{n^{2}}{4}
$$

Пусть $1 \geqslant x>x_{1}$. Тогда

$$
\left|P_{n-2}(x)\right| \leqslant \frac{1}{n} \sum_{i=1}^{n-1} \frac{U_{n-1}(x)}{x-x_{i}}=\frac{1}{n} U_{n-1}^{\prime}(x) \leqslant \frac{1}{n} U_{n-1}^{\prime}(1)=\frac{n^{2}-1}{3}
$$

так как максимум модуля производных полинома Чебьшева на отрезке $[-1,1]$ достигается только на концах отрезка и равен правой части неравенства (1) [1, с. 286]. Для $-1 \leqslant x<x_{n-1}$ доказательство аналогично.

ЛЕмма 2. Пусть для полинома $P_{2 n-2}(x)$ выполнены неравенства

$$
\begin{gathered}
0 \leqslant P_{2 n-2}\left(x_{i}\right), \quad x_{i}=\cos \frac{i \pi}{2 n} \quad(i=1,2, \ldots, 2 n-1), \\
\max _{x \in[-1,1]}\left|\left(1-x^{2}\right) P_{2 n-2}(x)\right| \leqslant 1 .
\end{gathered}
$$

Тогда справедливо неравенство

$$
\max _{x \in[-1,1]}\left|P_{2 n-2}(x)\right| \leqslant n^{2} .
$$

ДокАЗАТЕЛЬСтво. Для $x \in\left[x_{2 n-1}, x_{1}\right]$ доказательство такое же, как и в лемме 1. Пусть $x \in[-1,1] \backslash\left[x_{2 n-1}, x_{1}\right]$ и $P_{2 n-2}(x)>0$. Тогда

$$
\begin{aligned}
P_{2 n-2}(x) & =\frac{1}{2 n} \sum_{i=1}^{2 n-1}(-1)^{i-1} \frac{\left(1-x_{i}^{2}\right) P_{2 n-2}\left(x_{i}\right) U_{2 n-1}(x)}{x-x_{i}} \\
& \leqslant \frac{1}{2 n} \sum_{i=1}^{n} \frac{\left(1-y_{i}^{2}\right) P_{2 n-2}\left(y_{i}\right) U_{2 n-1}(x)}{x-y_{i}} \\
& \leqslant \frac{1}{2 n} \sum_{i=1}^{n} \frac{U_{2 n-1}(1)}{1-y_{i}}
\end{aligned}
$$


где $y_{i}=\cos (2 i-1) \pi / 2 n(i=1,2, \ldots, n)$.

Пусть $z_{i}=\cos (i \pi / n)$. Тогда

$$
\begin{aligned}
\frac{1}{2 n} \sum_{i=1}^{n} \frac{U_{2 n-1}(1)}{1-y_{i}} & =\frac{1}{2 n}\left\{\sum_{i=1}^{2 n-1} \frac{U_{2 n-1}(1)}{1-y_{i}}-\sum_{i=1}^{n-1} \frac{U_{2 n-1}(1)}{1-z_{i}}\right\} \\
& =\frac{1}{2 n}\left\{U_{2 n-1}^{\prime}(1)-\frac{U_{2 n-1}(1)}{U_{n-1}(1) U_{n-1}^{\prime}(1)}\right\} \\
& =\frac{1}{2 n}\left[\frac{2 n\left(4 n^{2}-1\right)}{3}-\frac{2 n\left(n^{2}-1\right)}{3}\right]=n^{2} .
\end{aligned}
$$

Пусть $P_{n^{2}-2}(x)<0$. Тогда

$$
\begin{aligned}
\left|P_{2 n-2}(x)\right| & =-P_{2 n-2}(x)=-\frac{1}{2 n} \sum_{i=1}^{2 n-1}(-1)^{i-1} \frac{\left(1-x_{i}^{2}\right) P_{2 n-2}\left(x_{i}\right) U_{2 n-1}(x)}{x-x_{i}} \\
& \leqslant \frac{1}{2 n} \sum_{i=1}^{n-1} \frac{U_{2 n-1}(1)}{1-z_{i}}=\frac{n^{2}-1}{3} .
\end{aligned}
$$

Лемма доказана.

3. Оценки норм производных полиномов на кубе в пространствах $\mathbb{R}_{l_{1}}^{m}$ и $\mathbb{R}_{l_{2}}^{m}$. Пусть $X$ и $Y$ - банаховы пространства и $\mathscr{L}(X, Y)$ - пространство непрерывных линейных отображений $X$ в $Y$. Введем обозначения

$$
\underbrace{\mathscr{L}\left(\mathbb{R}_{l_{i}}^{m}, \mathscr{L}\left(\mathbb{R}_{l_{i}}^{m}, \ldots, \mathscr{L}\left(\mathbb{R}_{l_{i}}^{m}, \mathbb{R}\right) \ldots\right)\right)}_{k \text { раз }}=\mathscr{L}_{i}^{k} \quad(i=1,2),
$$

$\nabla P_{n}(\widehat{x}), \nabla^{k} P_{n}(\widehat{x})$ - производные полинома $P_{n}(x)$ соответственно первого и $k$-го порядка в точке $\widehat{x}$.

Теорема. Пусть полином $P_{n}(x)$ вида (2) на кубе $Q$, где $Q=\left\{x \in \mathbb{R}^{m}\right.$ : $\left.-1 \leqslant x_{i} \leqslant 1, i=1,2, \ldots, m\right\}$ удовлетворяет неравенству $\left|P_{n}(x)\right| \leqslant 1$, тогда для его производных при $x \in Q$ справедливы следующие неравенства

$$
\begin{aligned}
\left\|\nabla P_{n}(x)\right\|_{\mathscr{L}_{2}^{1}} & \leqslant n^{2}, \\
\left\|\nabla^{2} P_{n}(x)\right\|_{\mathscr{L}_{1}^{2}} & =\max _{1 \leqslant i, j \leqslant m}\left|\frac{\partial^{2} P_{n}(x)}{\partial x_{i} \partial x_{j}}\right| \leqslant \frac{n^{2}\left(n^{2}-1\right)}{3} .
\end{aligned}
$$

ДоказАтЕльство. Из определения $\mathscr{L}_{i}^{k}$ следует, что $\mathscr{L}_{2}^{1}=\left(\mathbb{R}_{l_{2}}^{m}\right)^{*}=\mathbb{R}_{l_{2}}^{m}$, $\mathscr{L}_{1}^{1}=\left(\mathbb{R}_{l_{1}}^{m}\right)^{*}=\mathbb{R}_{\frac{m}{m}}$, где $\mathbb{R}_{\frac{m}{m}}$ - пространство с нормой $\|x\|_{m}=\max _{1 \leqslant i \leqslant m}\left|x_{i}\right|$. Равенство в левой части (6) нетрудно доказать из определения нормы оператора [4]. Пусть $\widehat{x}=\left(\widehat{x}_{1}, \widehat{x}_{2}, \ldots, \widehat{x}_{m}\right) \in Q$ и $\left|\widehat{x}_{i_{1}}\right|=\max _{1 \leqslant i \leqslant m}\left|\widehat{x}_{i}\right|$. Пусть $l-$ отрезок прямой $\left\{k_{1} x_{i_{1}}, k_{2} x_{i_{1}}, \ldots, k_{m} x_{i_{1}}\right\}\left(x_{i_{1}} \in[-1,1]\right)$, проходящий через точку 0 и $\widehat{x}$ $\left(k_{i}=\widehat{x}_{i} / \widehat{x}_{i_{1}}\right)$ и принадлежащий $Q$. Введем замену переменных $x_{i}=\cos \nu_{i}$. Тогда при $i \neq j$ из неравенства Бернштейна следует неравенство

$$
\left|P_{n \nu_{i} \nu_{j}}^{\prime \prime}(x)\right|=\left|\sin \nu_{i} \cdot \sin \nu_{j} P_{n x_{i} x_{j}}^{\prime \prime}(x)\right| \leqslant n(n-1) .
$$


Для $x \in l\left|x_{i}\right| \leqslant\left|x_{i_{1}}\right|$, поэтому из (7) вытекает неравенство $\mid\left(1-x_{i_{1}}^{2} P_{n x_{i} x_{j}}^{\prime \prime}(x) \mid \leqslant n(n-1), \quad x \in\left\{k_{1} x_{i_{1}}, k_{2} x_{i_{1}}, \ldots, k_{m} x_{i_{1}}\right\} \quad\left(x_{i_{1}} \in[-1,1]\right)\right.$.

В силу леммы 1 из (8) следует (6), так как при $i=j$ неравенство (6) следует непосредственно из (1).

Для доказательства неравенства (5) потребуется обобщенное неравенство Бернштейна, полученное в работе [5]. Приведем его формулировку.

Пусть $V$-центрально-симметричный выпуклый компакт в $\mathbb{R}_{l_{2}}^{m}, V^{*}$-полярамножества $V$. Для $z=\left(z_{1}, z_{2}, \ldots, z_{m}\right) \in \mathbb{C}^{m}$ положим

$$
\|z\|_{V}=\sup _{t \in V^{*}}\left|\sum_{i=1}^{m} t_{i} z_{i}\right| \text {. }
$$

Целая функция $f(z)$ имеет степень $v$, если для каждого $\varepsilon>0$ найдется $A_{\varepsilon}$ такое, что

$$
|f(z)| \leqslant A_{\varepsilon} \exp \left\{(1+\varepsilon)\|z\|_{V^{*}}\right\}
$$

для каждого $z \in \mathbb{C}^{m}$. Множество всех целых функций степени $v$, ограниченных на $\mathbb{R}^{m}$, обозначается $B(V)$. Пусть $\sigma>0$ и $g \in B(\sigma V)$. Тогда имеет место неулучшаемое неравенство

$$
\sup _{x \in \mathbb{R}_{l_{2}}^{m}}\|\nabla g(x)\|_{\mathbb{R}_{l_{2}}^{m}} \leqslant \sigma \frac{\operatorname{diam}(V)}{2} \sup _{x \in \mathbb{R}_{l_{2}}^{m}}|g(x)|,
$$

где $\operatorname{diam}(V)=\max _{x, y \in V}\|x-y\|_{\mathbb{R}_{l_{2}}^{m}}$.

Нетрудно проверить, что тригонометрический полином $P_{n}\left(\cos \nu_{1}, \ldots, \cos \nu_{m}\right)$ является целой функцией степени $n E$, где $E$ - шар радиуса единица в пространстве $\mathbb{R}_{l_{1}}^{m}: E=\left\{x \in \mathbb{R}^{m}: \sum_{i=1}^{m}\left|x_{i}\right| \leqslant 1\right\}$. Диаметр шара $E$ в метрике $l_{2}$ равен 2, поэтому из (9) для полинома $P_{n}(x)$ следует неравенство

$$
\sum_{i=1}^{m}\left[\sin \nu_{i} P_{n x_{i}}^{\prime}(x)\right]^{2} \leqslant n^{2} .
$$

Из (10) для $x$, принадлежащего отрезку $l$, следует неравенство

$$
\left(1-x_{i_{1}}^{2}\right) \sum_{i=1}^{m}\left[P_{n x_{i}}^{\prime}(x)\right]^{2} \leqslant n^{2} .
$$

В силу леммы 2 из (11) следует (5). Теорема доказана.

В дальнейших следствиях предполагается, что $\left|P_{n}(x)\right| \leqslant 1$ при $x \in Q$.

СледСТВИЕ 1. Для полинома $P_{n}(x)$ при $x \in Q$ справедливы неравенства

$$
\begin{gathered}
\left\|\nabla^{k} P_{n}(x)\right\|_{\mathscr{L}_{2}^{k}} \leqslant n^{2}(n-1)^{2} \cdots(n-(k-1))^{2}, \\
N_{k}(x)=\left\|\nabla^{k} P_{n}(x)\right\|_{\mathscr{L}_{1}^{k}}=\max _{x \in Q}\left|\frac{\partial^{k} P_{n}(x)}{\partial x_{1}^{i_{1}} \cdots \partial x_{m}^{i_{m}}}\right| \quad\left(i_{j} \geqslant 0, \sum_{j=1}^{m} i_{j}=k\right) .
\end{gathered}
$$

$\Pi p u k=2 p$

$$
N_{k}(x) \leqslant(1 / 3)^{p} \prod_{i=0}^{p-1}(n-2 i)^{2}\left((n-2 i)^{2}-1\right)=M_{2 p}
$$

npu $k=2 p-1 \quad N_{k}(x) \leqslant M_{2 p}(n-2 k)^{2}$. 
ДОКАЗАТЕЛЬСТВО следует из последовательного применения теоремы к полиному $\left\langle\nabla^{k} P_{n}(x),\left[h_{1}, h_{2}, \ldots, h_{k}\right]\right\rangle$, его градиенту $\left\langle\nabla^{k+1} P_{n}(x),\left[h_{1}, h_{2}, \ldots, h_{k}\right]\right\rangle$ или гессиану $\left\langle\nabla^{k+2} P_{n}(x),\left[h_{1}, h_{2}, \ldots, h_{k}\right]\right\rangle$. Здесь $h_{j}(j=1,2, \ldots, k)$-произвольные фиксированные нормированные векторы из $\mathbb{R}_{l_{i}}^{m}, k \geqslant 1$.

СледСтвие 2. Пусть $A$ - линейный оператор из $\mathbb{R}_{l_{i}}^{m}$ в $\mathbb{R}_{l_{i}}^{m}(i=1,2)$, $T=A Q, y=A x, x \in Q$. Пусть $B_{n}(y)$ - полином вида $(2) u\left|B_{n}(y)\right| \leqslant 1 n p u$ $y \in T$. Тогда для полинома $B_{n}(y)$ справедливо неравенство

$$
\left\|A^{*} \nabla B_{n}(y)\right\|_{\mathscr{L}_{i}^{1}} \leqslant n^{2}
$$

и если $А$ невьрождено, то

$$
\left\|\nabla B_{n}(y)\right\|_{\mathscr{L}_{2}^{1}} \leqslant\left(\min _{1 \leqslant j \leqslant m} \lambda_{j}\right)^{-1 / 2} \cdot n^{2}
$$

әде $\lambda_{j}$ - собственные значения матрицы $A A^{*}$.

Подобные неравенства можно распространить и на последующие производные, учитывая следствие 1.

СлеДСтвиЕ 3. Пусть при $x \in Q \quad\left|P_{2 n}(x)\right| \leqslant 1$ и для фиксированных $i, j$ $(i \neq j)$

$$
\frac{\partial^{2} P_{2 n}(x)}{\partial x_{i} \partial x_{j}} \geqslant 0
$$

Тогда

$$
\max _{x \in Q} \frac{\partial^{2} P_{2 n}(x)}{\partial x_{i} \partial x_{j}} \leqslant 2 n^{3}(2 n-1) .
$$

КБ "Аметист"

Поступило 16.01 .95

\section{СПИСОК ЦИТИРОВАННОЙ ЛИТЕРАТУРЫ}

1. Бернштейн С. Н. Собрание сочинений. Т. 2. М.: АН CCCP, 1954. 2. Kellog O. D. // Math. Zeit. 1927. V. 27. № 1. P. 55-66. 3. Don R. // J. Approx. Theory. 1974. V. 11. № 3. 4. Канторович Л.В., Акилов Г. П. Функциональный анализ в нормированных пространствах. М.: Физматгиз, 1959. 5. Ганзбург М. И. // УМH. 1979. Т. 34. № 1. С. 225-226. 